\title{
Supporting the Cross-cultural Appreciation of Traditional Chinese Puppetry Through a Digital Gesture Library
}

\author{
SHICHAO ZHAO, DAVID KIRK, and SIMON BOWEN, Open Lab, Newcastle University \\ DAVID CHATTING, Department of Design, Goldsmiths, University of London \\ PETER WRIGHT, Open Lab, Newcastle University
}

\begin{abstract}
In recent years, digital cultural heritage has attracted much attention in the HCI domain, but there are currently few studies that focus on enhancing the appreciation of intangible cultural heritage content amongst cross-cultural audiences. This article reports on the development of a Digital Gesture Library to support cross-cultural appreciation of traditional Chinese puppetry. We describe fieldwork with professional puppeteers to understand their practices and art form, which informed the development of the Digital Gesture Library, which uses a three-perspective archive of puppetry gestures and a tangible interface to support cross-cultural audiences' appreciation of puppetry and encourages further exploration of Chinese culture. We present findings on the efficacy of the Digital Gesture Library from qualitative and quantitative user studies and, from this, discuss the opportunities and challenges for developing digital technology for cross-cultural appreciation of intangible heritage.
\end{abstract}

CCS Concepts: • Human-centered computing $\rightarrow$ User studies; $\bullet$ Applied computing $\rightarrow$ Performing arts;

Additional Key Words and Phrases: Intangible cultural heritage, traditional Chinese puppetry, cross-cultural appreciation, digital gestural library, interaction design

\section{ACM Reference format:}

Shichao Zhao, David Kirk, Simon Bowen, David Chatting, and Peter Wright. 2019. Supporting the Cross-cultural Appreciation of Traditional Chinese Puppetry Through a Digital Gesture Library. ACM f. Comput. Cult. Herit. XX, X, Article XX

(November 2019), 19 pages.

http://dx.doi.org/10.1145/3341882

\section{INTRODUCTION}

Globalisation has penetrated many developing countries at an economic as well as a cultural level. A UNESCO report highlights that traditional cultures and arts, which have previously been passed down through generations, are under increasing threat [1]. Traditional Chinese puppetry is one such art form and was listed in the national nonmaterial cultural heritage list in 2006 [2]. Puppetry is a common form of opera performance within traditional Chinese theatre, and similar to other traditional Chinese opera, it has faced challenges because of its

Authors' addresses: S. Zhao, Open Lab, Newcastle University 1 Science Square, Science Central Newcastle Upon Tyne, NE4 5TG; email: s.zhao11@newcastle.ac.uk; D. Kirk, David.Kirk@newcastle.ac.uk; S. Bowen; email: simon.bowen@ncl.ac.uk; D. Chatting; email: david@davidchatting.com; P. Wright; email: p.c.wright@ncl.ac.uk.

Permission to make digital or hard copies of all or part of this work for personal or classroom use is granted without fee provided that copies are not made or distributed for profit or commercial advantage and that copies bear this notice and the full citation on the first page. Copyrights for components of this work owned by others than ACM must be honored. Abstracting with credit is permitted. To copy otherwise, or republish, to post on servers or to redistribute to lists, requires prior specific permission and/or a fee. Request permissions from permissions@acm.org.

(c) 2019 Association for Computing Machinery.

1556-4673/2019/11-ART28 $\$ 15.00$

http://dx.doi.org/10.1145/3341882

ACM Journal on Computing and Cultural Heritage, Vol. XX, No. X, Article XX. Publication date: November 2019. 
use of language and dialect that is not accessible to modern and cross-cultural audiences [3]. While research has been done to explore whether detailed context and character information or providing English/Mandarin subtitles makes puppetry more accessible, in general, the outcomes were not successful [4]. One aspect of Chinese puppetry that has been less well explored is gestures and the non-verbal communication of meaning and emotion. Chinese puppetry is rich in gestural and movement-based semiotics that are derived from Chinese rural culture and local opera. These also present challenges to modern cross-cultural audiences [5], but as a non-verbal form of communication, we wished to explore whether they are more amenable to digital support to give audiences a deeper and more complete understanding of the storylines and characters in puppetry performances [6].

In recent years, digital support for cultural heritage has begun to be explored in the HCI research community [7-12]. There has been significant discussion of the role of interactive technologies in enhancing support of visitors' engagement with cultural heritage settings and artefacts [13-15]. Extensive investigation and collaboration with audiences and heritage stakeholders has offered researchers new understandings of digital potential in exhibitions [16, 17] and has explored the development of audience engagement and participation $[18,19]$. In this article, we are seeking new ways to integrate gestural movements into a digital archive to help improve not only the accessibility of Chinese Puppetry to audiences but also encourage audiences' further exploration of its Chinese cultural background.

To this end, we developed an interactive system called the Digital Gesture Library. The design of the Digital Gesture Library was guided by insights from prior fieldwork and interviews with professional puppeteers. We also used a mixture of questionnaires, focus-groups, and workshops to specifically support and promote reflection upon aspects of the audience experience. Specifically, our findings explore how the Digital Gesture Library used: characteristic gestures as "ways in" to appreciating complex performances; a dynamic interface and threeperspective view to enable users to examine gestures in detail; a card-based tangible interface to support users in appreciating multiple gestures and their place in performance narratives; and physical Chinese puppets as part of the interface to deepen appreciation and encourage further exploration via sensory experience. We conclude by reflecting upon the opportunities and challenges for transferring the insights from our design and user studies of the Digital Gesture Library with traditional Chinese puppetry to other intangible cultural heritage settings, as a contribution to the $\mathrm{HCI}$ community.

\section{RELATED WORK}

Here, we focus specifically on several interactive digital systems intended to support the appreciation and engagement of puppet shows.

\subsection{Digital Technology for Puppetry Appreciation}

As early as 1998 , motion capture systems were designed to transfer puppetry gestures into digital/virtual form [20]. One of the design aims emphasised improving the appreciation of puppet shows, with the users' emotional expression being one of the main entry points used in some case studies [21, 22]. For instance, Fing AR was an interactive system that aimed to use a complete social pretend play experience to enhance children's emotional self-expression and understanding of puppetry [23]. However, the overly simplified gestures and basic emotional expression seems to have limited the cultural understanding of puppet shows; therefore, systematically displaying far more complex gestures in the system could create a far richer experience for its users. Another case study known as Emo Puppet used an interactive physical puppet used to support children while they were learning to understand emotions [24]. Whilst using emotions to enhance children's puppetry appreciation is worthwhile, a large number of audiences of Chinese traditional puppet shows are adults, and there is therefore an opportunity to explore how puppet gestures support deeper cultural appreciation in adult audiences. 
Some research projects have focused more on using digital animations to support the understanding of traditional puppetry stories. For instance, based on the photon mapping method, Zhu and colleagues developed a prototype that turned the traditional shadow plays into electronic forms that provided the audience with an immersive storytelling experience [25]. They offered delicate lighting effects and realistic martial arts sequences to reproduce the classic Chinese folk story "The Butterfly Lovers." However, the digital animation was solely focused on the reproduction, and the subtle meaning of the shadow play gestures were therefore not well displayed. The background story, dialogue, and traditional music of "The Butterfly Lovers" did not display with interpretation that would support users' appreciation. Another shadow play animation system exploited RRTConnect algorithm to capture the characteristics of motion from traditional Chinese shadow play then display the emulational animation for audiences $[26,27]$. The animation systems recreated realistic shadow play character movements to create an aesthetically pleasing environment for the audience. However, most of these research projects have focused on delivering experiences to users of the same or similar cultural backgrounds or who already appreciate the aesthetics of puppetry [28]. In these projects, the cultural relevance of a puppetry narrative and the in-depth meaning of puppetry gestures (movements) in this respect were less effectively interpreted by the audience [29, 6]. Expressing emotions through interactive technology is one valuable way of increasing audiences' appreciation of puppetry, but there is another important opportunity for interactive technology to help audiences in bridging cultural differences that have been less thoroughly explored. In particular, digital systems using gestures may enhance cross-cultural audiences' appreciation of puppetry and its deeper relationships with Chinese culture.

\subsection{Interactive Systems for Puppetry Performance}

A number of interactive systems have been developed that enable users to create digital puppets and digital puppetry performances. For instance, Cutout Animation is a video-based puppetry system that allows users to quickly create cutout-style animations by performing the character motions [30]. Meanwhile, digital storytelling systems are also being used-e.g., Shadow Story allows users to build digital puppets by themselves and then attempt to perform various scripts with the puppets whilst following the traditional aesthetic of the shadow plays [31]. Also, PUPPET is a digital system that allows children to play multiple roles in the creation of an interactive narrative [32]. Shi and colleagues combined the characteristics of Chinese folk culture with gesturebased interaction to design a digital shadow puppet. They developed an interactive system that exploited Kinectbased interaction and sensors to capture the children's movements to support them in creating their shadow play together [33]. Wan and colleagues also exploited Kinect-based to support users personally manipulation of the shadow characters with their gestures [34]; however, the gestures from the users were quite different from the gestures of traditional shadow play.

In these research projects, the interactive systems gave users greater autonomy and the capacity to exercise their individual creativity (such as making their own puppetry story). Further, users could experience simple puppetry movements via the digital puppets. For instance, Liang and colleagues developed series of systems that exploited deep motion sensor device to support the interactive animation and game applications of traditional shadow play [31]. However, cultural appreciation (such as the cultural meaning of gestures and stories) of the puppetry was not well supported as most of these research projects were entertainment-oriented. Furthermore, system interactions in user engagements and actual operation of the puppets have visible distinctions, and the potential for supporting audiences in engaging in the traditional puppet performance was not well explored in these research projects.

Our review of related work on digital technology for puppetry appreciation and puppetry performance, outlines an opportunity for further research on the points that our work explores. First, to investigate how digital technology might use gesture to support cross-cultural understanding. Second, to investigate how such digital technology can support audiences' appreciation of the physical aspects of puppetry performances. Here, we 


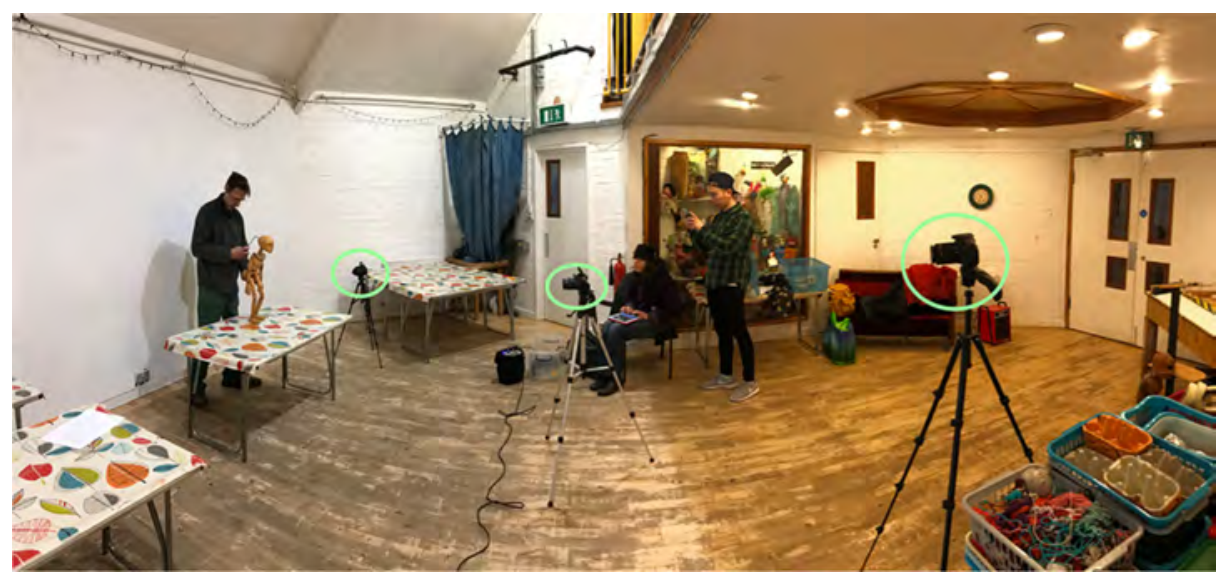

Fig. 1. The overall scene of the recording.

hypothesize that enhancing the in-depth appreciation and understanding of the gestural aspects of puppetry can close the gap between cross-cultural audiences and traditional Chinese puppetry [4]. We therefore framed the main research question for the study reported in this article as follows: How can interactive digital systems, based on puppetry gestures, be used to support cross-cultural audiences' appreciation of and further interest in traditional Chinese puppetry?

\section{FIELDWORK AND DATA COLLECTION}

Before designing any initial prototypes, we conducted fieldwork and collected puppetry gestures with professional puppeteers from Chinese and British puppetry theatres [35]. During the fieldwork, we introduced our research to participants and invited them to contribute to it through workshops and discussions. We obtained permission to create a video library of puppet gestures and make this available to participants and to the general public along with information about the puppeteers and theatres. To collect puppetry gestures that supported the Non-Chinese audiences' cross-cultural understanding, we collaborated with several British puppeteers who possessed abundant experience of performing traditional Chinese puppetry in Europe. During the puppetry fieldworks in $\mathrm{N}$ city (UK), we filmed with staff from $\mathrm{G}$ Theatre, a British visual theatre company that uses images, puppet animation, movement and live music to create theatrical productions of artistic excellence. As professional puppeteers, the staff of $G$ Theatre engaged in the filming process, which we chose to carry out in the theatre itself. The puppeteers possessed plenty of overseas performing experience, as well as having received some professional training in traditional Chinese puppetry. While collecting data, we intended to record puppetry gestures from different practitioners, hence deciding to contact $S$ city Theatre Academy (China), which offers a cademic s tudy in the "Performance of Traditional Chinese P uppetry." B elow, w e p rovide m ore detail about data collection in both settings.

\subsection{Filming in G Theatre, $\mathrm{N}$ city}

We used three cameras (a Nikon D3200 and two Canon EOS 600Ds) to capture puppetry movements as effectively as possible (Figure 1). The three cameras were set up to record three different perspectives: (i) the movements of the puppet, (ii) the gestures of the puppeteers, and (iii) the overall scene of the puppet and puppeteer (see Figure 2(b)). The content of the videos was determined through discussions with two professional puppeteers. We offered some initial thematic prompts for a performance, and then the puppeteers decided for themselves 

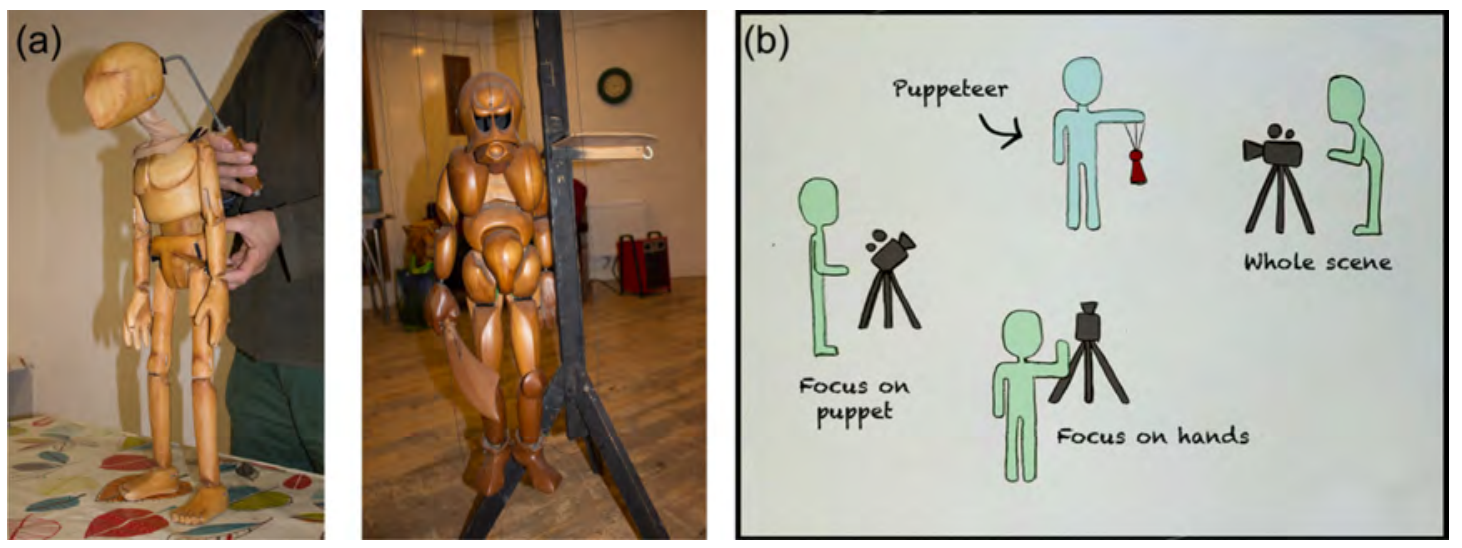

Fig. 2. (a) Tabletop puppet and Marionette. (b) Map of positioning.

how they would respond. For this recording, we chose two types of puppets, a tabletop puppet, and a marionette. After discussions with the puppeteers, we recorded their performance.

First, we recorded a passage of performance that includes the expression of different emotions. For a tabletop puppet (see the left one in Figure 2(a)), we did two individual puppeteer and two double puppeteer recordings, which included: (1) puppeteers demonstrating a very simple interaction with one tabletop puppet to deliver a series of emotions, and (2) puppeteers cooperating with each other to control one puppet to deliver a series of emotions from the puppet. For a marionette puppet (see the right on in Figure 2(a)), we made a single recording in which puppeteers demonstrated a very simple interaction to deliver a series of emotions.

We then recorded emotional and behavioural gestures separately, for the recording of the emotions, we captured three groups of movements from the tabletop puppet, which was found to be more appropriate for the performance of the emotions themselves. Taking advice from the puppeteers, we decided to capture a set of sequences of three emotions, which were: (1) happy, excited, and sadness; (2) shy, anxiety, and anger; and (3) laugh, shock, and cry. Then, we went on to conduct the recording of the behaviours of each tabletop puppet in this part. These two groups included: (1) get up, sad walking, and happy walking; and (2) jump, dance, and fly. Third, we outlined and conducted another three groups for the behaviours of the marionette: (1) get up, sad walking, and happy walking; (2) Jump, dance, and fly; and (3) get ready, sliding, and fighting.

To support the audiences understanding of the movements of British puppetry, we also recorded the movements that focused specifically on a puppet's legs in the third phase. This was to gain insight into the subtlety and intricacy of puppetry movements.

\subsection{Filming in S city Theatre Academy China}

For our second field site, our recording locations were set in the puppetry studio within $\mathrm{S}$ city Theatre Academy. We also used three cameras (three Canon EOS 5D Mark III) to capture three perspectives (see Figure 3(b)). In consideration of the puppets' outfit potentially covering the lens of the cameras when the puppeteers are manipulating the puppets, we changed the hand recording to the right side to ensure the quality of the recording was of a good standard. Again, we chose two types of puppets for this recording: (simple) traditional staffhead puppets demonstrated by puppetry students, and (more complex) string/wire-hung puppets (traditional marionettes) demonstrated by one of S city Theatre Academy's own puppetry lecturers. All of the content in this recording was discussed with the puppeteers to clarify the names of each gesture, which were based on their instructional system for puppeteers. 

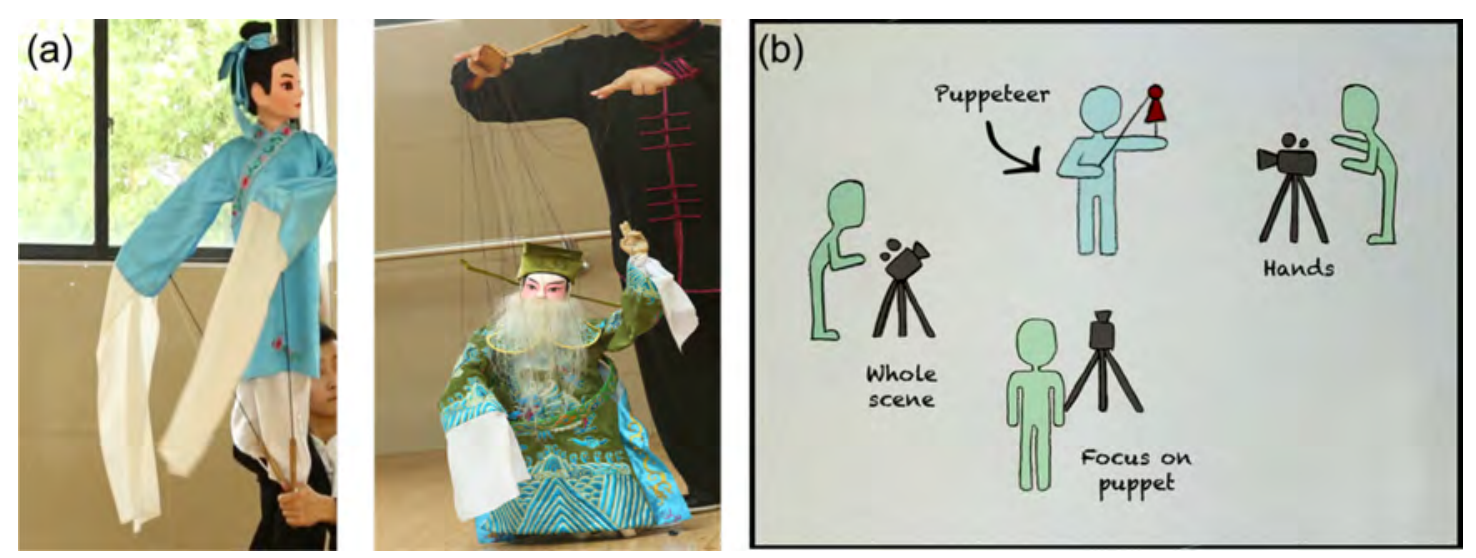

Fig. 3. (a) String/wire puppet and Marionette. (b) Map of positioning.

First, we recorded the gestures of the Chinese staff-head puppets (Figure 3(a)). We combined the discussion from the puppeteers and a professional instructional system to select a series of gestures for a digital library. All of the gestures in this phase were performed by two Chinese puppetry students who were then currently doing their undergraduate study. The gestures we recorded are different from the previous recording, as all of these gestures derive from opera puppetry, which has a strict standard of performance. We kept the original Chinese names alongside the English names to support a more accurate and concise understanding of the original meaning. The gestures we recorded in this phase were: Flipping the sleeve; Cloudy hands; Hand on sleeve; Scavenging ragged clouds; Rest the arms on the hips; One hand in front and one behind; Twisting of the hand; Young man's steps; Older man's steps; Black cloth's (Tsing Yi) steps; Male character (Huanglian) steps; A flag following the wind; Fast-paced step; Resting one arm on the hips; Placing one hand behind; and Cast sleeve.

Second, we recorded gestures from a string/wire-hung puppet/marionette (Figure 3(a)), which is the most complex type of Chinese traditional puppet. We recorded four kinds of gestures: (1) Breathe (inhale and exhale); (2) Raise a foot and move it; (3) Arms Movement; and (4) Flipping a sleeve to reveal a hand. The gestures performed were recorded by a puppetry lecturer from S city Theatre Academy, and during the recording, he explained roughly what was happening to support the understanding of each gesture. The gestures are focused more towards the movements of the arms and legs.

We also recorded a demonstration and step-by-step approach of the Chinese puppetry performance, during the puppetry course while the lecturer was teaching his students. This potentially supported us to gain an in-depth understanding of the characteristic gestures in traditional Chinese puppetry.

\section{DIGITAL GESTURE LIBRARY SYSTEM}

Based on the fieldwork and data collected, we designed and developed the Digital Gesture Library, an archive that displays Chinese traditional puppet shows as linear multi-perspective videos (puppetry gestures, puppet movement, and the whole performance) and multi-dimensional videos (gestures, genres, information of theatre, and puppeteers). This prototype uses a gestural archive to support audiences with varied cultural backgrounds in crossing cultural barriers (e.g., language, dialect, and local background). It also allows participants to appreciate the gestures of Chinese puppetry and engages participants to interact with a multimedia system. The application is composed of two parts: (1) a multimedia system and (2) a tangible interface. 

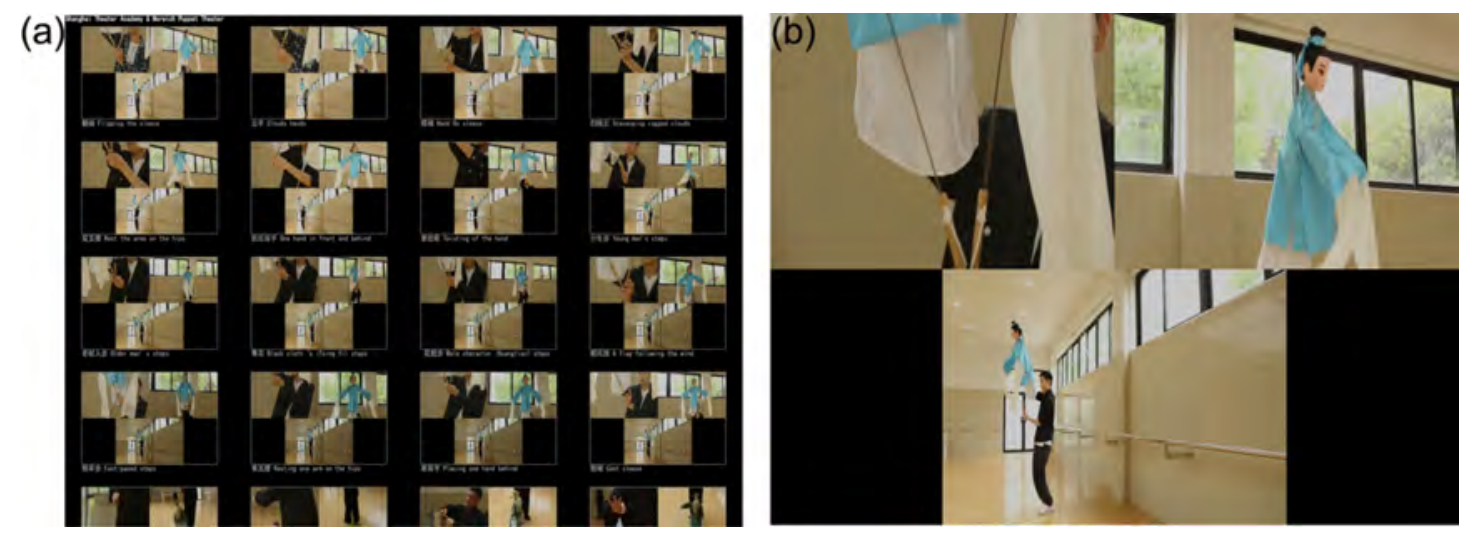

Fig. 4. (a) The main interface (sub-groups) of the Digital Gesture Library. (b) Three different perspectives of the gestures.

\subsection{Multimedia System}

The recorded gestures and performance details were integrated into a multimedia system, which can be browsed by means of an application. The entire multimedia infrastructure (composed of videos, images, and texts) was developed on an OS X system. The system has been implemented using standard, well-supported technologies. This guarantees that the entire digital infrastructure is portable, simply replicable, and easy to install in different venues. In particular, it was structured as follows:

4.1.1 Navigation Interface. In the menu interface of the system, we provide a brief introduction to the gestures and categorised them into sub-groups, which include various types of video-recorded puppetry gestures (Figure 4(a)). A brief introduction offers clear directions for users to watch specific gesture videos. Meanwhile, we designed the display of the videos and provided three different perspectives, which are presented in a format that fits the entire screen for users to browse more effectively; therefore, by clicking on any video, you can browse through the three different perspectives (Figure 4(b)). When users hold the mouse cursor on any video, this will trigger an interaction and play a preview of the video for 5 seconds-we intended for this to support users in making correct and unambiguous choices when browsing through the system and allowing them to choose more diligently.

4.1.2 Individual Gesture View. The elements of both single gestures and grouped gestures were combined and placed onto a page where they could be viewed. Through discussions with the puppeteers during the fieldwork, we grouped various gestures together based on the order that they are usually performed; this provided a systematic experience and demonstration for users. In this page, the three different perspectives were presented, the movements of puppeteers, the gestures of puppets, and the overall scene of the performance. These three perspectives allowed users to have a vivid experience of the puppet shows and to view more details on the operation and interaction between the puppeteers and the puppets themselves. More importantly, the users could gain a direct understanding of how to match gestures with movements. Three videos were played simultaneously without interruption by default, but users could choose specific perspectives as a full screen view to watch the details of a specific gesture or movement more closely.

4.1.3 Gesture Duration and Repetition. The length of each gesture video is usually quite short, relative to a complete puppet show. Thus, we implemented a setting that would allow for the system to repeatedly play a video for 5 seconds by default, rather than offering a pause option for users. Meanwhile, users can also utilise the left and right arrow keys to both increase and decrease the range of speed the video is playing at, by increments of 1 second. This repetition enables users to explore each movement and gesture more in depth. 


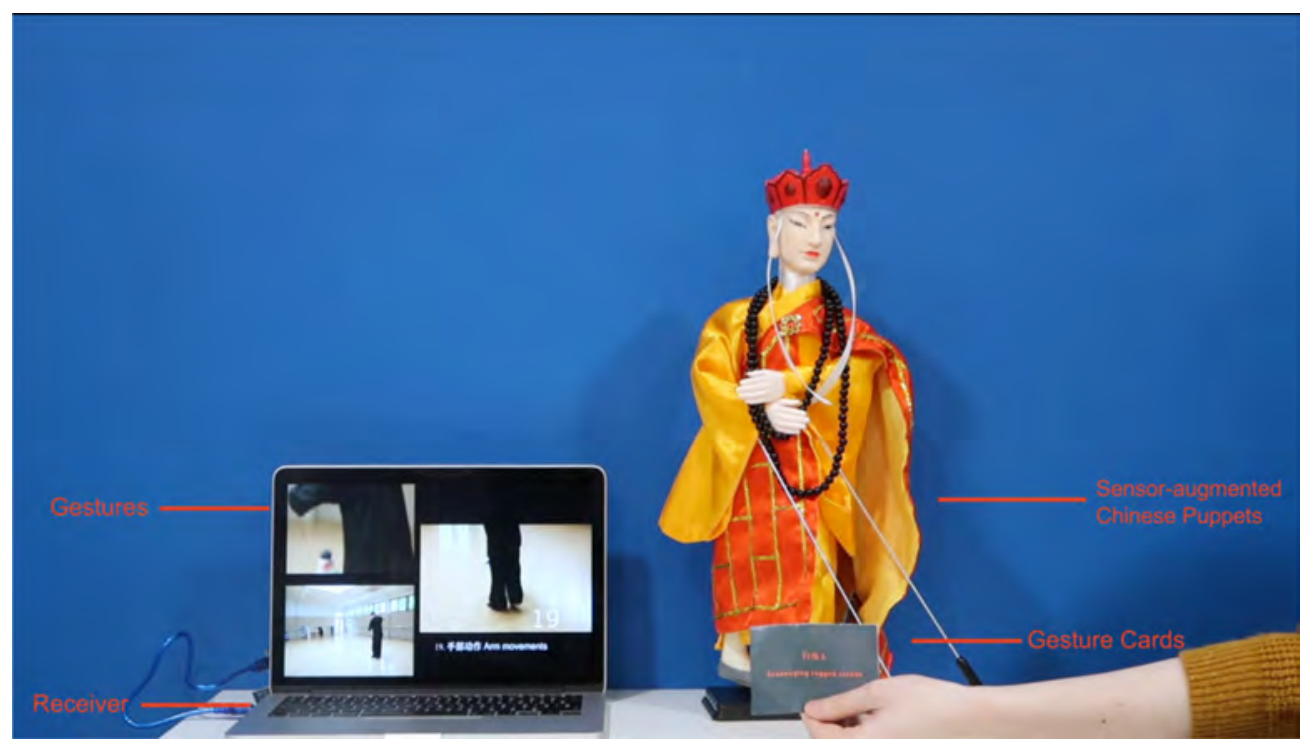

Fig. 5. Tangible puppet device.

\subsection{Tangible User Interface}

We developed a tangible interface for the Digital Gesture Library using Chinese calligraphy on physical cards and Chinese puppets. Our use of cultural artefacts was intended to offer users a more flexible means of interacting with the gesture library and another means of engaging with the related Chinese culture. The tangible user interface consisted of a set of digitally tagged gesture cards read by sensor-augmented Chinese puppets (Figure 5).

4.2.1 Digitally Tagged Gesture Cards. All of the gestures in the system were abstracted and illustrated with Chinese/English titles on palm-sized gesture cards. Each card/sensor matches with an appropriate video resource in the multimedia system for playback. We created a set of cards so that users could select, group, and rearrange them according to their developing interests. Every card contains a plastic digital tag that can connect and match with an appropriate video resource for playback. In the next paragraph, we discuss how gesture cards were used to interact with the puppet.

4.2.2 Sensor-augmented Chinese Puppets. We chose two classic puppet characters, Tang Monk (staff-head puppet) and Monkey King (string/wire-hung puppet) from the Chinese traditional folk story Journey to the West, for our tangible interface. These two puppets were hand-made in Zhangzhou, China. We placed an Arduino single board microcontroller inside the puppets that could interact with the gesture cards to connect to the gestural videos in the system. When the participants held a gesture card and touched the outfit of the puppet, the system played a corresponding gesture that matched with the gesture card. When a gesture cards is moved away, the video will stop playing. Participants randomly chose different $c$ ards to interact $w$ ith $t$ wo Chinese traditional puppets, as well as to watch gestural videos.

\section{USER STUDY}

Following our design of the Digital Gesture Library, we conducted user studies with the system. Our main research questions were: (1) How does the Digital Gesture Library enable cross-cultural users to understand Chinese puppetry? and (2) How could such digital technology encourage cross-cultural audiences to further explore 
Chinese puppetry? To address these questions, we deployed the Digital Gesture Library in a public setting to obtain quantitative data on user experience; and conducted a series of workshops with cross-cultural stakeholders, to gather qualitative data on their engagement with Chinese puppetry through the system.

\subsection{Methods}

We used a mixture of both qualitative and quantitative approaches, to support us in exploring system usability of the Digital Gesture Library and participants' cultural understanding of Chinese puppetry using digital technology [35, 36]. We utilised experience prototypes and interviews with focus groups, adopting an ExperienceCentred Design (ECD) [37] perspective, which enabled us to capture qualitative data on cross-cultural appreciation and engagement. This study was undertaken in three separate parts by the lead author from January to May 2018, across five European cities. The three activities include: (1) deploying the system in a public area and gathering users' responses via questionnaires; (2) a workshop and in-depth interviews with puppetry amateurs about their understanding of Chinese puppetry; and (3) a workshop with cross-cultural puppetry amateurs using the Digital Gesture Library and Chinese traditional puppets for storytelling.

\subsection{Recruitment}

For the public deployment, we installed the Digital Gesture Library in an entertainment room in a student accommodation building. 62 visitors completed questionnaires (28 male and 34 female); $42 \%$ of the visitors were undergraduate students, $37 \%$ were college students, and $21 \%$ were postgraduate students. In the puppetry workshop and interviews, we recruited five puppetry amateurs as participants (2 British, 2 Spanish, and 1 British-Chinese) split in to two groups for the workshop, ranging in age from 18 to 32 years. In the storytelling workshop, 6 participants ( 4 male and 2 female, 5 British and 1 Portuguese) took part. The storytelling workshop participants were allocated into three groups: (1) two British/Portuguese students in a university; (2) two British artists who worked in a gallery; and (3) a British middle-aged couple. All of the visitors who attended the public deployment were random visitors. All workshop participants described themselves being interested in puppetry, with five stating that they had experienced puppetry previously. However, none of the participants had experience with traditional Chinese puppet shows. We hoped to analyse diverse users who had different cultural backgrounds, age groups, and proficiencies, which would therefore provide valuable critical accounts for us to explore.

\subsection{Procedure}

5.3.1 Public Deployment. Average visitor attendance in the entertainment room was approximately 8 people per day during the working week and 15 people per day at the weekend, with the total amount of approximately 80 visitors in 7 days. We used a TV screen (1080p resolution, 16:9 aspect ratio) to display the interface of the Digital Gesture Library. A Chinese traditional puppet and elemental gesture cards were placed on the table. Figure 6 shows the original sketches of the installation layout. The questionnaire used nine questions, and out of these nine questions, three covered the usability of the system (intuitiveness, ease of use and responsiveness), another three questions focused on the idea of feedback on the use of the interactive device and interactive engagement (vision, touch, and interaction), and another three questions touched upon the quality of the digital content (clarity, understanding, and continuity of interest). Answers were given on a Likert scale [38] to analyse and rank the questions based on a four-level symmetric and balanced agree-disagree scale. More specifically, the adopted scale (strongly disagree, disagree, agree, and strongly agree).

5.3.2 Puppetry Workshop and Interviews. In this activity, five participants were invited to attend a 15-minute introductory session. The main purpose of our research was introduced, as well as the video data collection and the operation of the Digital Gesture Library. Through this introductory session, participants experienced the system and browsed each video resource in the system. Then, we demonstrated four videos of Chinese 


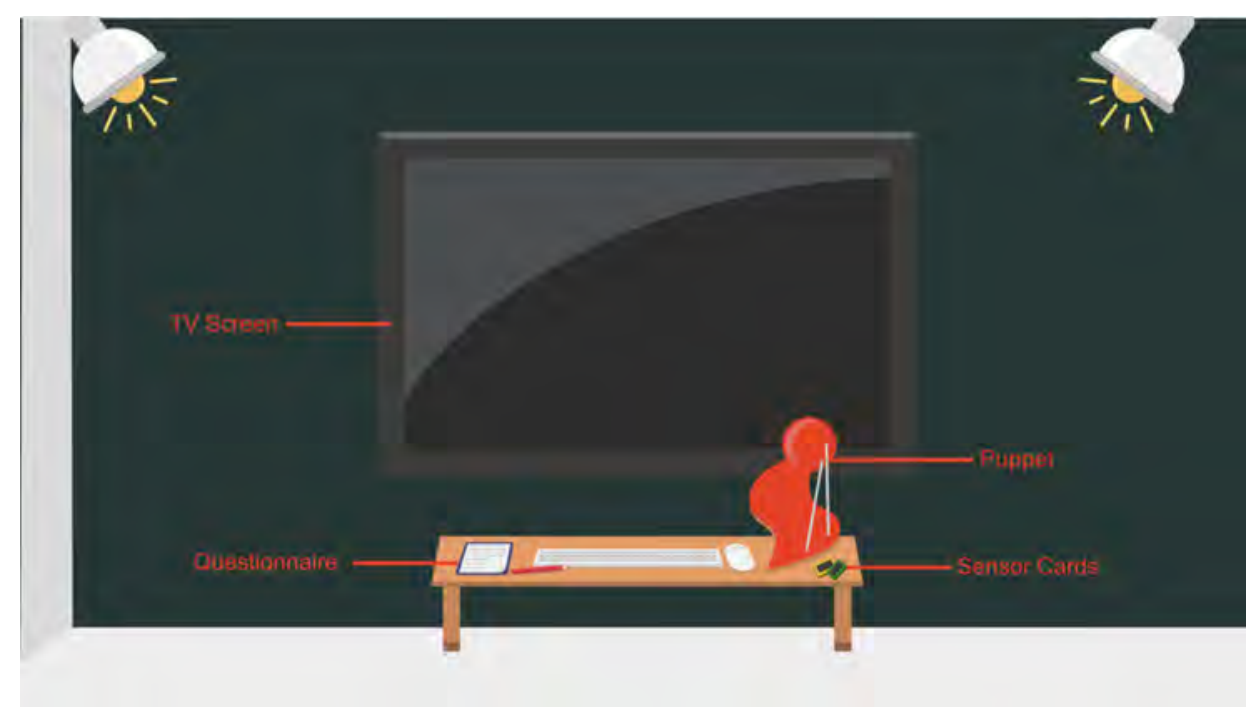

Fig. 6. The original sketches of the scheme deployed.

puppet shows directly on a laptop (not via the Digital Gesture Library) for participants, which lasted roughly 30-35 minutes. We also conducted in-depth interviews on their understanding of the videos.

5.3.3 Storytelling Workshop. In this activity, participants created their own stories through the Digital Gestural Library. Each participant was provided with a brochure that uses images and descriptions to ensure that participants understood the Digital Gesture Library (see Figure 7). As a group, two participants joined in with the engagement. Different gesture cards with names of elements, such as movements, types and emotions, had been offered to the participants to allow them to experience the Digital Gesture Library. Through discussion of their own or traditional stories, participants were able to talk about and construct a puppet show script. Meanwhile, two Chinese traditional puppets were provided to participants so that they could use two puppets to make a short play (approximately 20-30 mins) by combining their previously discussed stories that they had discussed prior. This story would connect with their own previous understandings rather than what they had gained from the Digital Gesture Library. After the workshop, we conducted an in-depth interview with each group.

\subsection{Data Analysis}

The data we had collected consisted of 62 questionnaire responses, collected from the public deployment; 10 hours of audio data recorded during the two workshops; and 2 hours of video data from the storytelling workshop and participants' brochure sketches during the two workshops. First, we analysed the questionnaire data in an attempt to evaluate the usability of the Digital Gesture Library. Second, from the interviews, we generated codes from the audio data relating to participants' understanding of Chinese puppetry and the Digital Gesture Library. Third, we analysed the video data and brochure notes as supplementary interaction notes to further understand how participants interacted with the Digital Gesture Library, as well as how participants interacted with other participants during the experience. The audio and video data were analysed through using Thematic Analysis [39]. All themes were supported by data excerpts. Initial codes were generated and refined using iterative analysis to produce coherent themes that were then further refined to establish useful findings. These codes were then used to recode the data, which was subsequently clustered to create a thematic structure for the data. All of the data was transcribed, with participants given a pseudonym for anonymity. Below, we discuss four main themes that emerged from the data. 

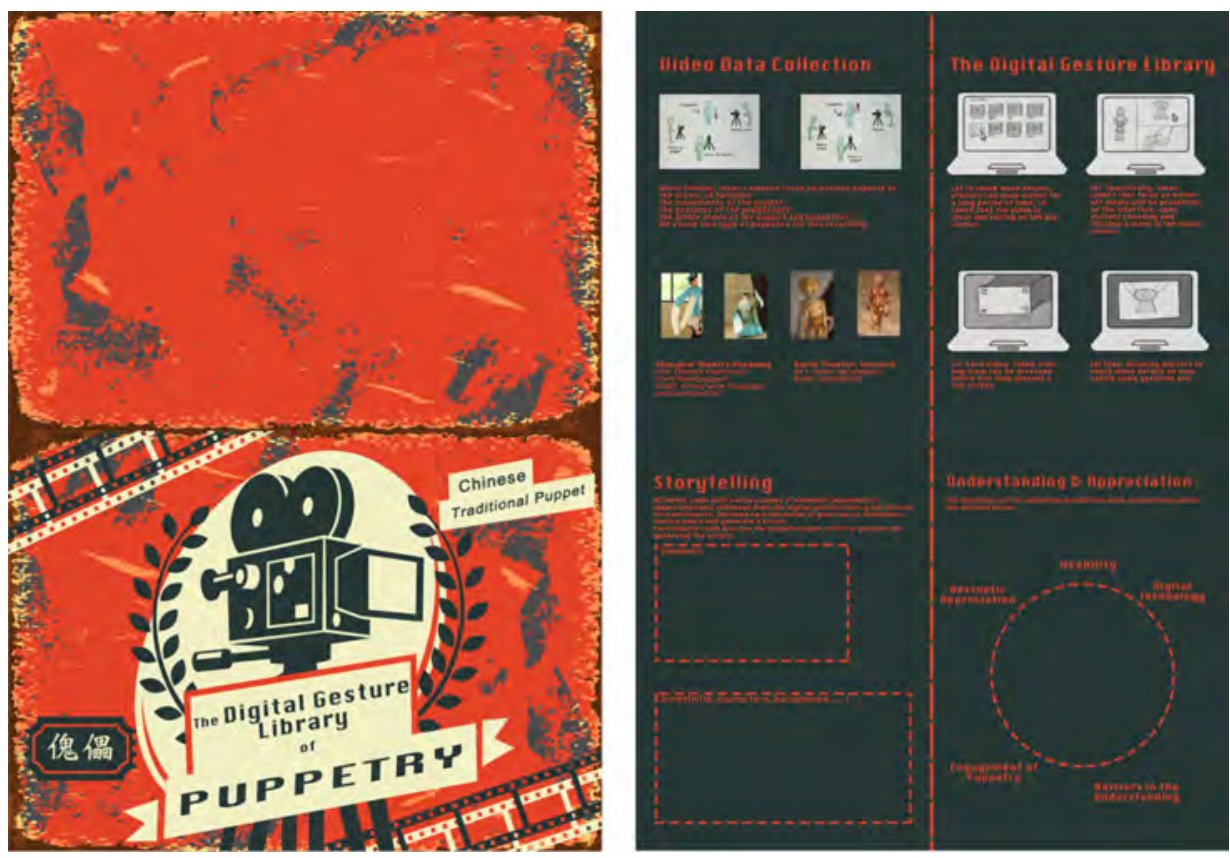

Fig. 7. The brochure was designed for a storytelling engagement workshop.

\section{FINDINGS}

Questionnaire responses suggest that the digital system was perceived as easy to use, intuitive, and responsive by most respondents. All of the questions in the questionnaires are shown in Figure 8 (System usability), Figure 9 (Interaction), and Figure 10 (Content). 77\% of respondents quickly understood the main purpose of the Digital Gesture Library (35\% agree, $42 \%$ strongly agree). A similar number (79\%) found the system responsive (50\% agree, $29 \%$ strongly agree). $85 \%$ rated the system easy to use (48\% agree, $37 \%$ strongly agree). Respondents rated digital content both clear (33\% agree, $51 \%$ strongly agree) and understandable (24\% agree, $46 \%$ strongly agree. These data confirm that visitors perceived the system as sufficiently usable. In the following sections, we explore in more depth some of the key features of the participants' experiences of using the Digital Gesture Library and some reflections on interactive technology to support the cross-cultural understanding of traditional Chinese puppetry.

\subsection{Characteristic Gestures as "Ways in" to Chinese Puppetry}

Traditional Chinese puppetry can seem mysterious to cross-cultural audiences. The particular focus on gestures within our Digital Gesture Library seem to be a useful "way in" or starting point from which users can decrypt an unfamiliar and seemingly complex cultural artform. During their experiences of the Digital Gesture Library, our participants expressed that elements such as the puppetry gestures or body language could be seen as a potential pathway in helping people to understand the cultural relevance of Chinese puppetry more precisely. Especially for non-Chinese participants, the elemental gestures provide them the clear relevance of puppetry movements. Here, Cameron had expressed his experience: "For me, prior to this, as a foreigner, I couldn't understand this at all. . . but everything is different now, I clearly know the meaning of the movements. Like some body language that's used in puppetry is not international. So, it might be some internal language that they use." 


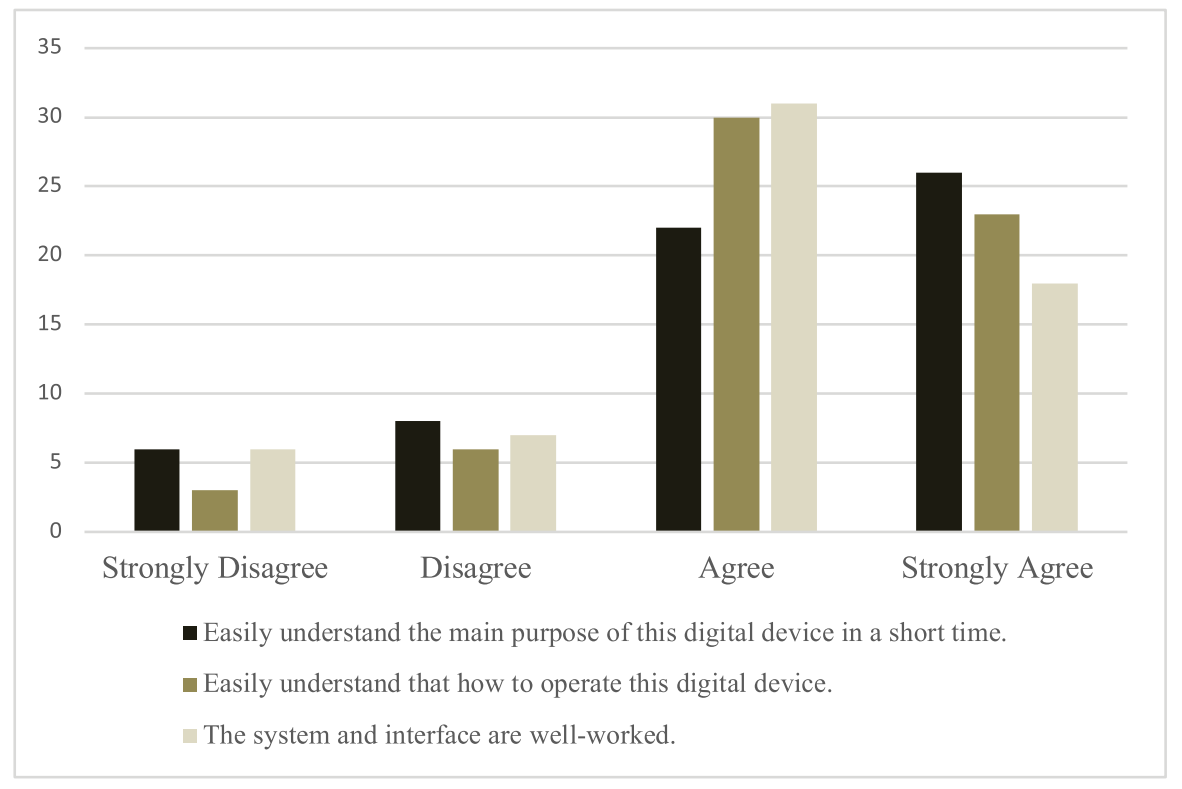

Fig. 8. The part of system usability of questionnaire.

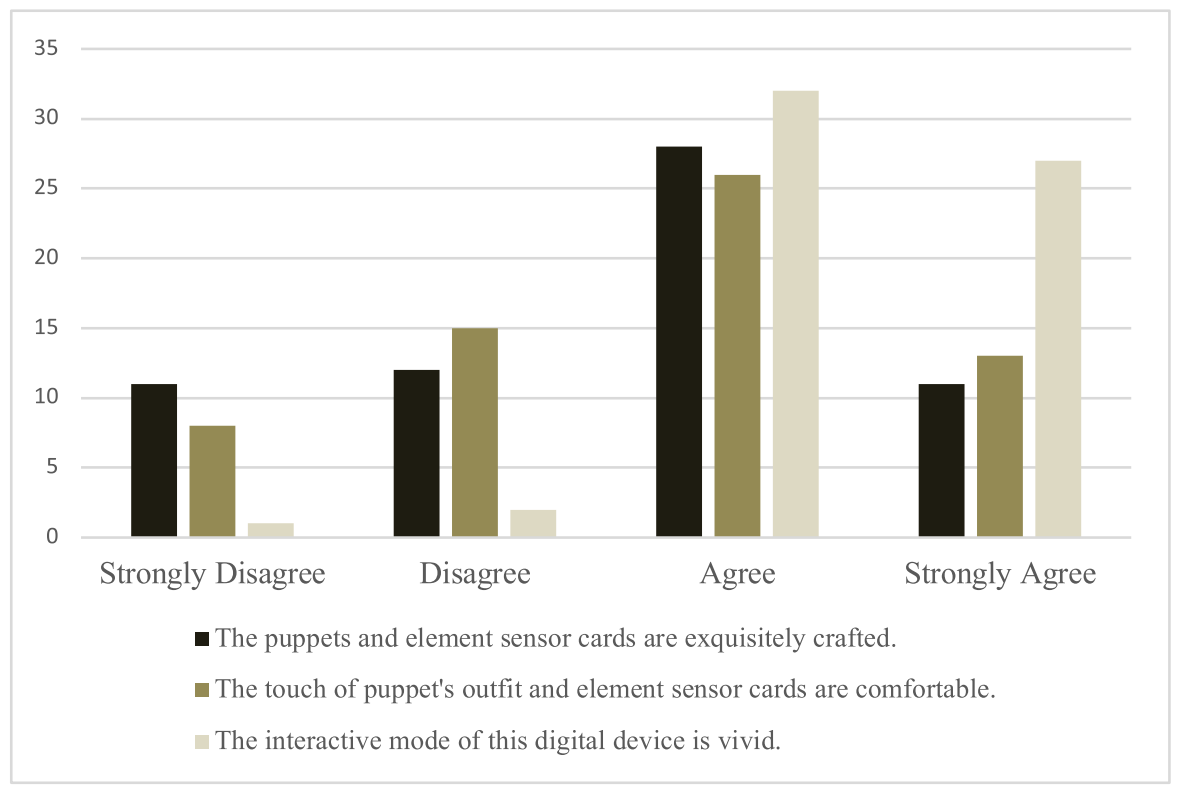

Fig. 9. The part of interaction of questionnaire.

The participants' understanding of the storyline was also supported by elemental gestures, which may potentially allow the non-Chinese audiences to have an enjoyable and fluent puppetry experience. For instance, Alex shared his thoughts: "These elements are like separated letters or words, which I can combine to be a sentence, then I can understand the story. Even though I can't speak Chinese, I still can understand this story." 


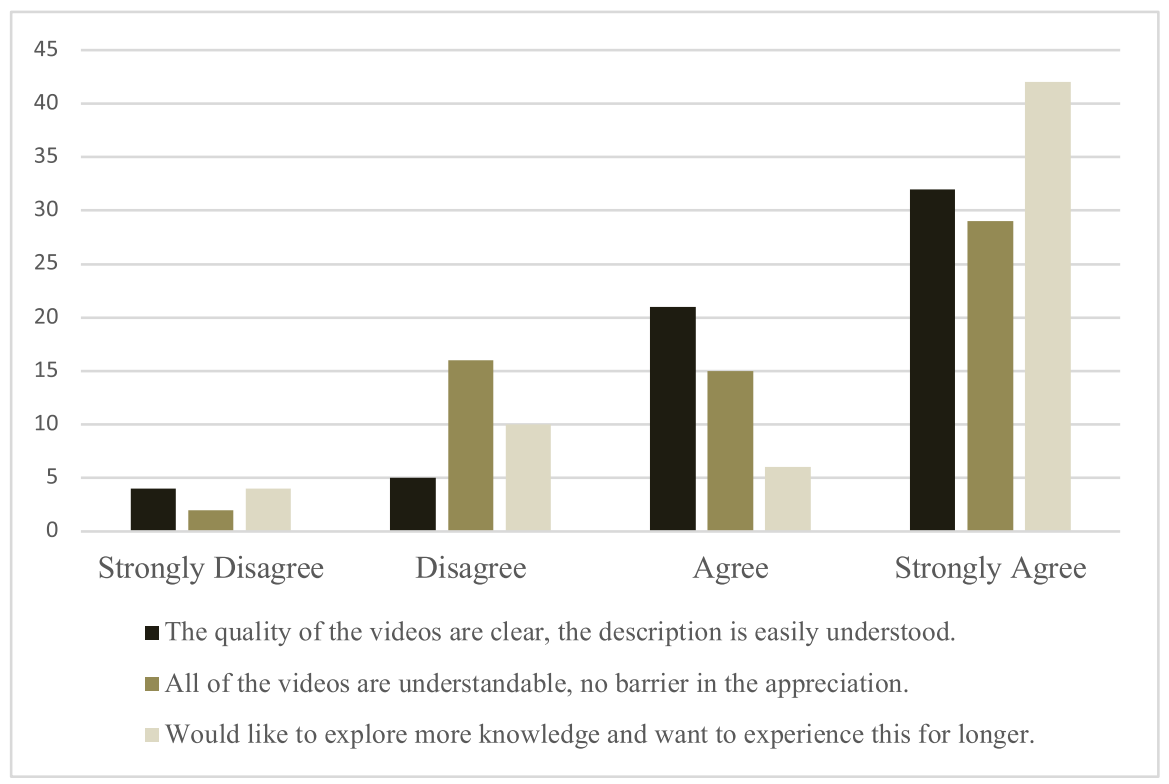

Fig. 10. The part of content of questionnaire.

Meanwhile, the focus of the participants' experience was changed by briefly observing the puppet's outfit and the background music, which enabled for an in-depth understanding of the emotions from puppet show to be gained, which effectively avoided participants from giving up on watching the puppet show, because they did not understand it. In Sarah's words: "I watched the Chinese puppet show before, just checked some nice outfit, and some noisy music, then I stopped the watching. Because I don't understand the dialogues, this makes me feel bored. But this time, after I observed the elemental gestures, I felt the emotions from the puppets and even from the puppeteers. I don't even remember how long I watched this show, I was really enjoying it."

\subsection{Decrypting Chinese Puppetry through Examining Gestures}

Participants' curiosity around Chinese traditional puppet shows was stimulated by their experience of viewing different perspectives of gestural videos. Some participants had expressed that they did not notice the gestures from the puppeteers themselves, similar to that of the interaction between the puppet and puppeteer in their previous experiences. However, it is the "secret" behind the puppet shows that the majority of the participants were genuinely interested in.

Our participants often discussed that the experience of the multi-perspective views provided a more comprehensive understanding of puppet shows. The attentions of the participants were not only focused on the performance of the puppets, in which Thomas mentioned: "I think it's the three perspectives as well on some of them, so from all the different perspectives it gives you an idea of how puppetry looks from all different angles and how you can do it from just looking at those different angles."

In the Digital Gesture Library, the three different perspectives from the three different camera positions can be switched randomly, and any video can go into a full screen mode, allowing participants to explore the details in specific videos, which enables them to concentrate more on a particular gesture or movement. This feature received a high level of praise from the participants. For the participants such as Paul, this type of interaction helped them to form a self-exploration approach to a puppetry experience: "I think it's a quite good function. I 
like how it gives you the option to have a look at three different screens. Especially, I can choose any viewpoint by myself, step by step, some of them I watched for so many times until I found the secret."

The ability to move between gestures, and to explore each gesture from three perspectives, enabled users to examine gestures in greater detail, which supported their process of "decryption." In this process, the participants' exploration was not limited to the gestures from puppets but also included observing how puppeteers manipulate the puppets themselves.

\subsection{Tangibility of Chinese Puppetry}

Different interactive approaches of the Digital Gesture Library encouraged participants to spend more time to explore the subtleness of the traditional Chinese puppetry.

6.3.1 Cards Support Interactions and Storytelling with Multiple Gestures. By analysing participants' feedback from the workshop, it is possible to understand that the expectation of the puppetry experience from participants was not only limited to simply watching the puppet show. Jade explained that she enjoyed reading the Chinese/English explanation on the cards, allowing her to gain a rough understanding of the gestures and create a sequence through the different elemental cards to record her progress: "With the library you could put lots of different videos together and make a story, like the randomisation of the cards. Read the meaning first. Also, when I used the cards it would remind me of what video you've watched so you can keep going back and forwards without forgetting what the movement was or what video it was that you were on."

The elemental gesture cards connected personal stories or emotions with the puppet show, as shared by Brian, who explains this type of interaction offered him an immersive experience: "I think after using the element cards and building my story I think it was quite fun. And just feel like I am being a part of a Chinese story."

6.3.2 Puppets Develop Understandings through Sensory Experiences. Holding the gesture card to touch the Chinese puppet had also been discussed on numerous occasions by participants, such as Alex who displayed interest when interacting with the puppet: "I prefer scanning it than actually going through the videos by themselves. Cos It's like I can touch the puppet and have an interaction with it. I haven't experienced this before."

Alternately, the puppet displays and interactions with it could also encourage the participants to closely explore the mechanisms and movements of the Chinese puppets themselves. The participants also enjoyed getting to know the more subtle details of the puppet, as Thomas described: "We think this puppet looks gorgeous, we observed how they were moving the arms, its different with my previous imagination. Not like just watching it, I never thought I could actually really play this."

\subsection{Moving Between the Parts, the Whole, and Elsewhere to Develop Understanding}

We found that $77 \%$ of the respondents expressed that they want to explore more knowledge after this experience ( $45 \%$ agree plus $32 \%$ strongly agree). During the workshop, we noticed that participants conducted their experience based on their personal understanding of the gestural elements. For instance, Thomas did not go straight to view the gestures in the digital library first, it was after he watched the puppet show that he began to experience the digital library for himself, and therefore the questions he was generating could be answered when using the library: "So, for me, the step one is you watch your puppet show and then step two is you use the library to recap the movements. Then following on from that maybe you re-watch the puppet shows so you can understand the dialogue and what's happening."

Moreover, from the interaction within Group 3, participants searched the different relevant elements of the gestures initiatively through the comparison to explore and discuss the in-depth meaning: Sarah paused the video of the "Flipping the sleeve" gesture, and began watching the "Hand on sleeve" and "Cast sleeve" gesture.

From this, she elaborated: "I compared those similar gestures, which was able to help me understand the accurate meaning, I think this is really important for understanding the whole plot of the story." 
From Group 2's interaction notes, we found that participants used their mobile devices to search online for more background information on Chinese puppet characters and Chinese traditional folk stories whilst they were using the digital library. These studies were based on the discussion they had during their engagement as a group, which guided the participants in exploring more aesthetic knowledge from a lower to higher scale: During the discussion, they used the internet to search Chinese traditional stories to get to know the background stories, and grasp some knowledge of the character of the puppets (workshop observation notes).

Meanwhile, when the participants from Group 1 were performing their own puppet show, they also combined their personal emotions and stories to match with the Chinese characters: Paul and fade researched Chinese folk music as background to help themselves in the cultural atmosphere (workshop observation notes).

Their understanding of the puppet show was then not only limited to the gestural meaning but also extended to relevant Chinese cultural background and interpretations: "We found a very common example of Chinese folk music to use as background music, we like to discover more relevant knowledge to make us more connected to the show, and we can get to know so much more Chinese traditional cultures at the same time."

After participants finished their engagement, we found that the participants did not stop their discussion with each other at this point, they recorded the elements and script for planning another spontaneous performance. Even though the engagement had finished, the participants' exploration of Chinese puppetry did not finish when the workshop did. Further activities from Group 1 were as follows: After they finished their performance, they still talked about their stories and immerse in the stories/emotions. Also, they talk about wanting to let their kids experience this to allow them to get to know Chinese traditional cultures (workshop observation notes).

\section{DISCUSSION}

Based on the findings from designing the Digital Gesture Library and studying its use, we now elaborate our contributions from this study to $\mathrm{HCI}$ and the digital heritage community.

\subsection{Reflections on the Puppetry Study}

Our design of the Digital Gesture Library was strongly informed by our fieldwork with puppetry stakeholders, which enabled us to incorporate their professional expertise in the contents of the archive and how it should be organised and interacted with. Specifically, we were advised by puppeteers on what significant and characteristic gestures to record and how to categorise them. This helped us gain an in-depth understanding of traditional Chinese puppetry that was faithful to the original purpose of the performance [10]. Likewise, it helped to support us in extracting characteristic elements accurately. With traditional Chinese puppetry being a form of gestural art, the puppetry gestures have played a crucial role in communicating cultural significance and traditional storylines [1]. Although some digital applications manage to increase the users' entertainment quite significantly, the performance and gestures themselves have become over-simplified. Between the design for entertainment-oriented puppetry and design for puppetry learning, we suppose that supporting cross-cultural audiences appreciation of and engagement with traditional Chinese puppetry could be a further design opportunity [40]. Thus, we designed the multiple viewpoints to most effectively communicate and make examinable characteristic gestures from puppets, puppeteers and performance scenes, as well as display these elements in a digital archive. Through the Digital Gesture Library, the participants conducted storytelling and self-expression through their own puppet shows, and explored the Chinese traditional culture this puppetry draws upon.

However, from our fieldwork, we only collaborated with a few puppeteers and were only able to collect a small number of gestural recordings. The characteristic videos only covered three types of traditional Chinese puppetry; consequently, this limited fieldwork restricted our findings to a certain extent. Moreover, there are further possibilities of multi-perspective viewpoints that could be designed and developed, for instance a perspective that allows for a first-person view, which would offer audiences a new experience of observing puppetry from the puppeteer's own point of view. We see this, however, as an explicit strength of the approach. Like many 
traditional qualitative research methods, there is value in idiographic case studies (detail over generalisability). The methods of our fieldwork and design, as well as the interactive technology we used and design, all constitute an additional form of critical reflection, embodied within the application we have extracted, designed and deployed, regardless of our findings.

\subsection{Digital Technology for Cross-Cultural Appreciation of Chinese Puppetry}

The main barriers in the cross-cultural audiences' appreciation of traditional Chinese puppetry include lack of familiarity with the Chinese language and the Chinese cultural background $[4,6]$. Although the gestures of the puppets themselves are derived from human movement, puppetry gestures have also been heavily influenced by Chinese traditional opera [22], which formed a unique system of Chinese puppetry. Therefore, a key question for our user study became: How does the Digital Gesture Library enable cross-cultural users to understand Chinese puppetry?

The Digital Gesture Library recorded the characteristics of gestures for different types of puppets, different characters or roles, and also different emotions and gestures. This offered participants a clear and concise understanding of the background knowledge. From the analysis of the questionnaires and workshops, we noticed that the participants had a clear understanding of the puppet show, and that they were able to retell the main storyline of the show they had seen. Our participants have also talked about their own thoughts on the subtle differences between puppets' emotional expressions rather than only having a rough experience of the story. We would say the gesture library goes some way to overcome the linguistic barrier experienced by cross-cultural audiences. In addition, participants were not only interested in the puppets' gestures but also in the interaction between the puppets and the puppeteers, plus the scenes and settings of the performance as a whole.

Compared to the traditional approach of watching a puppet show, the three-perspective views offered participants the opportunity to explore the mystery of the puppet show by themselves and gain a deeper understanding of the puppetry gestures. Furthermore, our participants expressed that through using the gestures cards to interact with sensor-augmented Chinese puppets that enabled them to act out a gesture associated to a card, and greatly aroused their interest in interacting with the puppets. The participants repeatedly mentioned that they enjoyed interacting with the puppet's outfit. This interaction encouraged them to be more curious when exploring the mystery of Chinese puppetry and allowed for participants to get closer in observing the subtleties of the puppets themselves (for example appearances, outfits, character, etc.). We believe that prior to participants engaging with the puppets, it would be more beneficial for them to interact with the puppets themselves to gain familiarity with puppetry and gestures [24]. This would enable for the gap between audiences and traditional puppetry to become more closed. Also, this tangible interface could be an entertaining function to engage users to experience the Digital Gesture Library.

Accordingly, analysing the cross-cultural participants experiences of the Digital Gesture Library, we found it offered effective help to participants who have different cultural backgrounds to overcome language barriers. Participants were also able to gain an in-depth understanding of the artistic knowledge of a puppetry performance. We would like to emphasise that extracting the multi-perspective gestures as an elemental library was not intended to reduce the complexity of cultural artforms into smaller pieces. We suppose that the Digital Gestural Library helps to identify key elements that express and characterise the complexity of cultural artform, whilst not exhaustively representing the culture itself.

\subsection{Digital Technology for Cross-cultural Appreciation of Other Relevant Intangible Cultural Heritage}

Our study of the Digital Gesture Library with Chinese Puppetry suggests that such digital technology could also support cross-cultural audiences in exploring the artistic subtleties of Chinese traditional cultures [41] and intangible heritage more broadly. There are many elements of traditional Chinese puppetry (e.g., movements, 
music, scripts) that originate from other forms of Chinese intangible cultural heritage such as folk stories, traditional opera gestures, and folk dance. In particular, for these intangible cultural heritage, non-linear video resources are frequently collected to safeguard cultural heritage, but there are plenty of video resources that are under-utilised [2, 42, 43]. As a standard method, video data collection has been used to safeguard the performative cultural heritage and the cultural skills from craftspeople [44, 45]. In this study, we recorded multiple perspectives to build an elemental gesture archive, and offered users the option to switch between the videos to view different perspectives of a gesture. Our study suggests that the use of digital technology to support the redesign of non-linear video resources could go further to support audiences' understanding. This broadens the possibilities for the usability and communicability of non-linear videos and also offers the specific design approach to combine Chinese traditional cultures and digital technology. For the re-editing of the previous video data and the future data collection work, the multiple perspectives viewpoints of gestures and movements could offer more possibility when it comes to supporting the understanding of audiences.

Furthermore, our study potentially suggests how interactive systems of this form could support cross-cultural audiences in crossing some of the cultural barriers during their appreciation, as well as further engaging their interests in this area. We hope that the general design idea (a digital archive to display multiple perspectives views of gestures) and the interface (gesture cards with tangible cultural artefacts) we used could also support the appreciation of and engagement with other similar intangible cultural heritages, specifically those of performative arts-based on gestures or movements (for instance, traditional dance and traditional opera) for cross-cultural audiences. From our interviews with participants, it was clear that engagement with the characteristic gestures would never replace the traditional performance of traditional Chinese puppetry in the theatre (and we would never advocate this). However, conversely, before cross-cultural audiences go to the theatre to watch the show, the characteristic gestures in a digital archive could offer them a comprehensive and in-depth understanding of the relevant culture. During their experiences with our system, the participants' understanding of the storyline throughout Chinese traditional fairy-tales and Chinese traditional cultures have improved remarkably. Digital technology, using multi-perspective archives of characteristic elements and relevant cultural artefacts in their tangible interfaces, could support cross-cultural appreciation of intangible cultural heritage in other settings.

\section{CONCLUSIONS}

We have described our Digital Gesture Library for supporting the cross-cultural appreciation of traditional Chinese puppetry and its relationship to fieldwork conducted with British and Chinese professional puppeteers. A subsequent user study encouraged participants to discuss and reflect upon their cultural experiences during their engagements with the Digital Gesture Library. Our aim through this study was to offer valuable transferrable insights into the design of digital technologies that support and stimulate the appreciation of intangible cultural heritage. The results of our study suggest that digital multi-perspective archives of gestures with tangible interfaces including relevant cultural artefacts do increase cross-cultural audiences' engagement with Chinese puppetry. Meanwhile, this study also opened an opportunity to design digital systems that use similar principles to increase cross-cultural audiences' appreciation of intangible cultural heritage and encourage their further exploration of the broader cultural context this heritage draws upon and expresses.

\section{REFERENCES}

[1] The Convention for the Safeguarding of the Intangible Cultural Heritage. 2003.UNESCO Press. https://unesdoc.unesco.org/ark:/48223/ pf0000132540.

[2] The State Council of the People's Republic of China. 2010. Strengthen the protection of Chinese "intangible cultural heritage." The State Council of the People's Republic of China Press. http://www.china.org.cn/china/2010-06/02/content_20171387_2.html.

[3] Zhifeng Wu. 2009. The research of digitalized technology of the puppet show with the motion capture technology. F. Univ. Electron. Sci. Technol. China 9 (2009), 6-7.

[4] Zhimin Xu and Xuefeng Xin. 2007. The Phylogeny of Chinese Puppet Show. Literature of Shandong Press.

[5] Frank Proschan. 1981. Puppet voices and interlocutors: Language in folk puppetry. F. Amer. Folklore 94 (374), 527-555. 
[6] Shichao Zhao, David S. Kirk, Simon Bowen, and Peter C. Wright. 2018. Enhancing the appreciation of traditional Chinese painting using interactive technology. Multimod. Technol. Interact. 2 (2018), 2.

[7] Laura A. Maye, Dominique Bouchard, Gabriela Avram, and Luigina Ciolfi. 2017. Supporting cultural heritage professionals adopting and shaping interactive technologies in museums. In Proceedings of the Conference on Designing Interactive Systems (DIS'17). ACM, New York, NY, 221-232. DOI:http://dx.doi.org/10.1145/3064663.3064753

[8] Barry Brown, Ian MacColl, Matthew Chalmers, Areti Galani, Cliff Randell, and Anthony Steed. 2003. Lessons from the lighthouse: Collaboration in a shared mixed reality system. In Proceedings of the SIGCHI Conference on Human Factors in Computing Systems (CHI'03). ACM, New York, NY, 577-584. DOI:http://dx.doi.org/10.1145/642611.642711

[9] Athanasios Gaitatzes, Dimitrios Christopoulos, and Maria Roussou. 2001. Reviving the past: Cultural heritage meets virtual reality. In Proceedings of the 2001 Conference on Virtual Reality, Archeology, and Cultural Heritage (VAST'01). ACM, New York, NY, 103-110. DOI:http://dx.doi.org/10.1145/584993.585011

[10] Marc Levoy, Kari Pulli, Brian Curless, Szymon Rusinkiewicz, David Koller, Lucas Pereira, Matt Ginzton, Sean Anderson, James Davis, Jeremy Ginsberg, Jonathan Shade, and Duane Fulk. 2000. The digital michelangelo project: 3D Scanning of large statues. In Proceedings of the 27th Annual Conference on Computer Graphics and Interactive Techniques (SIGGRAPH'00). ACM, New York, NY, 131-144. DOI:http: //dx.doi.org/10.1145/344779.344849

[11] Rafal Wojciechowski, Krzysztof Walczak, Martin White, and Wojciech Cellary. 2004. Building virtual and augmented reality museum exhibitions. In Proceedings of the 9th International Conference on 3D Web Technology (Web3D’04). ACM, New York, NY, 135-144. DOI:http: //dx.doi.org/10.1145/985040.985060

[12] Tim Hawkins, Jonathan Cohen, and Paul Debevec. 2001. A photometric approach to digitizing cultural artifacts. In Proceedings of the Conference on Virtual Reality, Archeology, and Cultural Heritage (VAST'01). ACM, New York, NY, 333-342. D0I:http://dx.doi.org/10.1145/ 584993.585053

[13] Dirk vom Lehn and Christian Heath. 2005. Accounting for new technology in museum exhibitions. Int. 7. Arts Manage. 7, 3 (2005), $11-21$.

[14] Eric Klopfer, Judy Perry, Kurt Squire, Ming-Fong Jan, and Constance Steinkuehler. 2005. Mystery at the museum: A collaborative game for museum education. In Proceedings of the Conference on Computer Support for Collaborative Learning: Learning 2005: the Next 10 years! (CSCL'05). ACM, New York, NY, 316-320.

[15] Laura A. Maye, Fiona E. McDermott, Luigina Ciolfi, and Gabriela Avram. 2014. Interactive exhibitions design: What can we learn from cultural heritage professionals? In Proceedings of the 8th Nordic Conference on Human- Computer Interaction: Fun, Fast, Foundational (NordiCHI'14). ACM, New York, NY, 598-607. DOI:http://dx.doi.org/10.1145/2639189.2639259

[16] Nick Taylor. 2014. Supporting community participation in interactive exhibits. In Proceedings of the International Symposium on Pervasive Displays (PerDis'14). ACM, New York, NY, 74. DOI:http://dx.doi.org/10.1145/2611009.2611031

[17] Kieran Ferris, Liam Bannon, Luigina Ciolfi, Paul Gallagher, Tony Hall, and Marilyn Lennon. 2004. Shaping experiences in the hunt museum: A design case study. In Proceedings of the 5th Conference on Designing Interactive Systems: Processes, Practices, Methods, and Techniques (DIS’04). ACM, New York, NY, 205-214. DOI:http://dx.doi.org/10.1145/1013115.1013144

[18] Christian Heath, Paul Luff, Dirk Vom Lehn, Jon Hindmarsh, and Jason Cleverly. 2002. Crafting participation: Designing ecologies, configuring experience. Visual Commun. 14, 1 (2002), 9-33.

[19] Jon Hindmarsh, Christian Heath, Dirk Vom Lehn, and Jason Cleverly. 2005. Creating assemblies in public environments: Social interaction, interactive exhibits and CSCW. CSCW F. 14, 1 (2005), 1-41.

[20] David J. Sturman. 1998. Computer puppetry. IEEE Comput. Graph. Appl. 18, 1 (1998), 38-45.

[21] William Dolby. 1978. The origins of Chinese puppetry. In Bulletin of the School of Oriental and African Studies, University of London 41. 97-120.

[22] Fan Pen Li Chen and Bradford Clark. 2010. A survey of puppetry in China. In Asian Theatre fournal 27. University of Hawai'i Press, 333-365.

[23] Zhen Bai, Alan F. Blackwell, and George Coulouris. 2015. Exploring expressive augmented reality: The FingAR puppet system for social pretend play. In Proceedings of the 33rd Annual ACM Conference on Human Factors in Computing Systems (CHI'15). ACM, New York, NY, 1035-1044. DOI:http://dx.doi.org/10.1145/2702123.2702250

[24] Jesús I. Martínez. 2014. emoPuppet: Low-cost interactive digital-physical puppets with emotional expression. In Proceedings of the 11th Conference on Advances in Computer Entertainment Technology (ACE'14). ACM, New York, NY. DOI:http://dx.doi.org/10.1145/2663806. 2663873

[25] Yi-Bo Zhu, Chen-Jia Li, I. Fan Shen, Kwan-Liu Ma, and Aleksander Stompel. 2003. A new form of traditional art: Visual simulation of Chinese shadow play. In Proceedings of the ACM SIGGRAPH Conference on Sketches and Applications (SIGGRAPH'03). ACM, New York, NY, 1-1. DOI:http://dx.doi.org/10.1145/965400.965458

[26] Shu-Wei Hsu and Tsai-Yen Li. 2005. Planning character motions for shadow play animations. In Proceedings of the Conference on Computer Animation and Social Agents (CASA'05). Vol. 5, 184-190. 
[27] Shu W. Hsu and Tsai-Yen Li. 2005. Generating secondary motions in shadow play animations with motion planning techniques. In Proceedings of the ACM SIGGRAPH Conference on Sketches and Applications (SIGGRAPH'05). ACM, New York, NY. DOI:http://dx.doi. org/10.1145/1187112.1187195

[28] M. Gail Hickey. 2012. Asian Indian celebrations of ethnicity: Perspectives from the mid-western united states. In International fournal of Intangible Heritage, Vol. 7. Jongno-gu, Seoul, 31-44.

[29] Elisa Giaccardi. 2011. Things we value. In Magazine Interactions. ACM, New York, NY, 17-21. DOI:http://dx.doi.org/10.1145/1897239. 1897245

[30] Connelly Barnes, David E. Jacobs, Jason Sanders, Dan B. Goldman, Szymon Rusinkiewicz, Adam Finkelstein, and Maneesh Agrawala. 2008. Video puppetry: A performative interface for cutout animation. In Proceedings of the ACM SIGGRAPH Asia Papers (Asia’08). ACM New York, NY. DOI:http://dx.doi.org/10.1145/1409060.1409077

[31] Fei Lu, Feng Tian, Yingying Jiang, Xiang Cao, Wencan Luo, Guang Li, Xiaolong Zhang, Guozhong Dai, and Hongan Wang. 2011. ShadowStory: Creative and collaborative digital storytelling inspired by cultural heritage. In Proceedings of the SIGCHI Conference on Human Factors in Computing Systems (CHI'11). ACM, New York, NY, 1919-1928. DOI:http://dx.doi.org/10.1145/1978942.1979221

[32] Paul Marshall, Yvonne Rogers, and Mike Scaife. 2002. Puppet: A virtual environment for children to act and direct interactive narratives. In Proceedings of the 2nd International Workshop on Narrative and Interactive Learning Environments. 8-15.

[33] Yan Shi, Fangtian Ying, Xuan Chen, Zhigeng Pan, and Jinhui Yu. 2013. Restoration of traditional Chinese shadow play-piying art from tangible interaction. F. Visual. Comput. Animat. 25, 1 (2013), 33-43.

[34] Bo Wan, Xiu Jun Wen, Lingling An, and Xiaoling Ding. 2015. Interactive shadow play animation system. Int. 7. Comput. Electric. Automat. Control Info. Eng. 9, 1 (2015), 127-132.

[35] Barney G. Glaser and Anselm L. Strauss. 1998. The Discovery of Grounded Theory: Strategies for Qualitative Research. Aldine Transaction Press.

[36] John W. Creswell. 2002. Research Design: Qualitative, Quantitative and Mixed Methods Approaches. SAGE Publications Press, London.

[37] Peter Wright, and John McCarthy. 2010. Experience-Centered Design: Designers, Users, and Communities in Dialogue. Morgan \& Claypool Press.

[38] Dane Bertram. 2015. Likert Scales Are the Meaning of Life. Topic Report Press.

[39] Virginia Braun and Victoria Clarke. 2008. Using thematic analysis in psychology. Qualitat. Res. Psychol. 3, 2 (2008), 7-101.

[40] Shichao Zhao. 2019. Exploring how interactive technology enhances gesture-based expression and engagement: A design study. Multimod. Technol. Interact. 2019, 3, 13

[41] Maria Bonn, Lori Kendall, and Jerome McDonough. 2016. Preserving intangible heritage: Defining a research agenda. In Proceedings of the 79th ASIS\&T Annual Meeting: Creating Knowledge, Enhancing Lives through Information \& Technology (ASIST'16). ACM New York, NY. DOI:https://dl.acm.org/citation.cfm?id=3017456

[42] Hui Liang, Shujie Deng, Jian Chang, Jian Jun Zhang, Can Chen, and Ruofeng Tong. 2016. Tong. Semantic framework for interactive animation generation and its application in virtual shadow play performance. In Proceedings of the 15th ACM SIGGRAPH Conference on Virtual-Reality Continuum and Its Applications in Industry. ACM, 137-145.

[43] Maria Teresa Linaza, Miriam Juaristi, and Ander Garcia. 2014. Reusing multimedia content for the creation of interactive experiences in cultural institutions. 3D Res. Chall. Cultur. Herit. 8355 (2014), 104-118.

[44] Shichao Zhao and David S. Kirk. 2016. Using interactive digital media to support transcultural understanding of intangible Chinese cultural heritage. In Proceedings of the CHI Conference Workshop-Involving the Crowd in Future Museum Experience Design.

[45] Shichao Zhao, David S. Kirk, Simon Bowen, and Peter C. Wright. 2019. Cross-cultural understanding of Chinese traditional puppetry: Integrating digital technology to enhance audience engagement. In International fournal of Intangible Heritage, Vol. 14. Jongno-gu, Seoul.

Received December 2018; revised March 2019; accepted June 2019 